\title{
Caracterização dos pontos de venda de bebidas alcoólicas no entorno de escolas estaduais
}

\author{
Classification of alcohol outlets in the area surrounding state \\ schools
}

Loren Milagros Salazar Cardoza (https://orcid.org/0000-0002-6889-8377) ${ }^{1}$

Diego Spinoza dos Santos (https://orcid.org/0000-0002-3107-7719) ${ }^{2}$

Doroteia Aparecida Hofelmann (https://orcid.org/0000-0003-1046-3319) ${ }^{3}$
${ }^{1}$ Programa de PósGraduação em Saúde

Coletiva, Universidade Federal do Paraná. R. Padre Camargo 280, $3^{\circ}$ andar, Alto da Glória. 80060-240 Curitiba PR Brasil. loren.scardoza@gmail.com ${ }^{2}$ Secretaria Municipal de Saúde. Curitiba PR Brasil.

${ }^{3}$ Programa de Pós-

Graduação em Saúde

Coletiva, Universidade

Federal do Paraná. Curitiba PR Brasil.

\begin{abstract}
The aim of this study was to characterize the environments of Curitiba's state schools in relation to commercialization of alcoholic beverages and investigate the association with local socio-economic indicators. A cross-sectional study in commercial establishments was carried out around 30 state-owned schools. We applied a survey to characterize the locations in relation to the availability of alcoholic beverages. Differentiation between school environments regarding income was estimated by multilevel mixed-effects model. We evaluated 200 commercial establishments, $66.0 \%$ commercialized alcohol. Of them, 52 (39.4\%) were off-premise alcohol outlets and $80(60.6 \%)$ on-premise. In on-premise category, neighborhood markets (16.5\%) and gas stations (10.1\%) had higher presence and half of schools had this type of this establishment in their environment. These establishment had more variety of alcoholic beverages and lower prices. In school's environments located in lower income's neighborhoods, the number of neighborhood market and bars were higher. It was observed the presence of establishments that commercialized alcoholic beverages and with attendance to the public during the classes period, near the evaluated schools.
\end{abstract}

Key words Alcoholic beverages, Commerce, Environment, School
Resumo Oobjetivo foi caracterizar o entorno das escolas estaduais de Curitiba em relação à comercialização de bebidas alcoólicas e investigar a associação com indicadores socioeconômicos dos locais. Trata-se de um estudo transversal em estabelecimentos comerciais do entorno de 30 escolas estaduais do município. Aplicou-se questionário para descrever as características dos locais em relação à disponibilidade de bebidas alcóolicas. Diferenças nos entornos das escolas de acordo com a renda foram estimadas por modelo de regressão multinível misto. Foram avaliados 200 estabelecimentos, 66,0\% comercializavam bebidas alcoólicas. Dos locais avaliados, $52(39,4 \%)$ foram pontos de venda para consumo de álcool no local, e 80 (60,6\%) para consumo fora do local. Nesta categoria, os mercados de bairro (16,5\%) e os postos de gasolina $(10,1 \%)$ tiveram maior presença e metade das escolas tinham esse tipo de estabelecimento em sua vizinhança. Esses locais apresentaram maior variedade de bebidas alcoólicas e menores preços. Nos entornos das escolas localizadas em bairros de menor renda, o número de mercados de bairro $e$ bares foi maior. Observou-se presença de estabelecimentos que comercializavam bebidas alcoólicas e com atendimento ao público durante o período de aulas nas proximidades das escolas avaliadas.

Palavras-chave Bebidas alcoólicas, Comércio, Ambiente, Escola 


\section{Introdução}

No Brasil, $50 \%$ dos adultos e $30,9 \%$ dos adolescentes de 13 a 17 anos consomem bebidas alcoólicas $^{1,2}$, um dos países na América Latina com maior consumo per capita nos adultos, superando a média mundial ${ }^{3}$. O consumo de álcool é um fator de risco para doenças crônicas e fator causal para mais de 200 enfermidades e agravos ${ }^{3}$, estando também relacionado à prática de sexo inseguro, acidentes veiculares e violência; além disso, $\mathrm{o}$ consumo precoce pode influenciar o tipo de consumo na vida adulta ${ }^{4,5}$.

Por essa razão, a Organização Mundial da Saúde tem incentivado a criação de políticas e investigações que versem sobre essa questão e contribuam para diminuição dos danos ocasionados pelo consumo de álcool, sobretudo nos grupos vulneráveis ${ }^{6}$. Entre as medidas com potencial de diminuir os danos associados ao consumo abusivo de álcool está a limitação da disponibilidade de bebidas alcoólicas?

São três os tipos das principais políticas de prevenção ao consumo abusivo de álcool: 1) disponibilidade econômica, considerada a mais eficaz, sobretudo para reduzir o consumo entre adolescentes, referindo-se às taxações que incidem nos preços das bebidas; 2) disponibilidade de varejo, que corresponde às facilidades de compra e acesso, diz respeito a estratégias como a delimitação dos pontos de venda, diminuição da densidade de estabelecimentos, idade mínima de compra, restrição de dias e horários, instituição de serviços de venda responsáveis, regulação de venda nos locais para consumo interno e implantação de um sistema de licenças; e 3) a disponibilidade social, relativa ao acesso de fontes que não o varejo do álcool ${ }^{8}$.

Entre essas três vertentes, aquela que tem se destacado nas investigações acadêmicas é a da disponibilidade de varejo, mensurada de forma física nas pesquisas por meio da densidade de estabelecimentos (número de pontos de venda em determinada área), sendo encontradas relações com consumo de álcool, violência e danos ${ }^{9,10}$.

O local tem papel importante nos comportamentos de saúde, e partindo de um enfoque preventivo, como já descrito, a redução da exposição e da disponibilidade de estabelecimentos que comercializam álcool é considerada uma forma de reduzir as taxas de consumo, pois preços maiores e distâncias mais longas diminuiriam o poder aquisitivo e o consumo por adolescentes ${ }^{11}$. Além disso, considerando que os adolescentes passam a maior parte do tempo nas escolas e que, no geral, elas refletem as características da vizinhança, algumas pesquisas investigaram o entorno escolar e comportamentos de saúde, encontrando associações e reforçando esse enfoque ${ }^{12-14}$.

Em revisão sistemática realizada por Bryden e colaboradores ${ }^{15}$, observou-se que a classificação empregada para avaliar os pontos de venda de álcool é relativa à disponibilidade de pontos de venda para consumo no local (on premise) e a pontos de venda para consumo fora do local (off premise). Na primeira categoria estão incluídos restaurantes, bares, cafés, lanchonetes e todos aqueles estabelecimentos que oferecem um lugar em suas dependências para que as bebidas alcoólicas. A segunda categoria contempla supermercados, mercados de bairro, mercearias, lojas de conveniência, postos de gasolina e, de forma geral, estabelecimentos que comercializam álcool para que seja consumido fora do local.

Alguns estudos encontraram relação entre o uso de álcool e suas consequências com aspectos como viver em uma comunidade organizada e com melhores características socioeconômicas $^{16-19}$. Porém, em relação à oferta de álcool, resultados de estudos mostraram maior densidade de pontos de venda de bebidas alcoólicas em bairros com condições socioeconômicas desfavoráveis, o que sugere maior exposição da população em maior vulnerabilidade social ${ }^{20,21}$.

A maioria das pesquisas foram realizadas em países de alta renda como Estados Unidos, Canadá, Inglaterra, Escócia, Austrália e Suíçaa ${ }^{11,22-26} \mathrm{e}$ com metodologias variadas. No país, são poucas as pesquisas encontradas que têm descrito ou avaliado a disponibilidade de álcool ${ }^{7,27}$ especialmente para adolescentes.

Conhecer as características da disponibilidade comercial de bebidas alcoólicas no território e investigar se existe alguma associação com variáveis socioeconômicas do local torna-se importante para efetuar a implantação e implementação de estratégias de saúde, políticas regulatórias de álcool e legislações para zoneamento e uso do solo.

São poucos os estudos desse tipo nos países em desenvolvimento ${ }^{7,27}$, o que corrobora a necessidade de realizar pesquisas na área, pois diferenças econômicas e culturais influenciam a sociedade e os resultados podem ser distintos. Essa importância é reforçada quando são consideradas as fontes de acesso dos adolescentes brasileiros - festas, amigos, mercados e família ${ }^{2}$-, bem como a facilidade de compra que alguns estudos têm relatado ${ }^{2,27,28}$, reforçada pelos resultados de pesquisas que avaliam a percepção de acesso dos 
adolescentes, que consideram ser fácil obter bebidas alcoólicas ${ }^{28-30}$.

O presente estudo tem como objetivo caracterizar os pontos de comercialização de bebidas alcoólicas no entorno das escolas estaduais de Curitiba e investigar a associação com indicadores socioeconômicos dos locais.

\section{Métodos}

Trata-se de um estudo transversal com coleta de dados primários que integra o projeto "Excesso de peso e características do ambiente escolar em estudantes de Curitiba - PR", cujo objetivo foi investigar a associação do excesso de peso com características socioeconômicas, demográficas, do ambiente escolar e comportamentos relacionados à saúde em adolescentes de escolas estaduais de da capital paranaense. As áreas descritas no presente trabalho correspondem aos entornos das escolas que participaram no projeto mencionado, ou seja, serão apresentados apenas dados dos entornos das escolas, não dos alunos.

Como já apontado, o estudo foi realizado no município de Curitiba, que é constituído por dez regiões administrativas e 76 bairros $^{31}$. A cidade é considerada a mais populosa do estado do Paraná, com população estimada, em $2016^{32}$, de 1.893.997 habitantes. De acordo com indicadores do Instituto de Pesquisa e Planejamento Urbano de Curitiba (IPPUC), é considerada 100\% urbanizada e seu Índice de Desenvolvimento Humano (IDH) para o ano de 2010 foi considerado alto $(0,823)$.

\section{Amostra}

O número de escolas a serem avaliadas foi definido a partir da amostra de alunos calculada para responder aos objetivos do estudo maior. Partimos do número de alunos das escolas estaduais (110.238) e empregamos os seguintes parâmetros: prevalência do desfecho de 50\%, margem de erro de quatro pontos, nível de confiança de $95 \%$, proporção de perdas esperadas, e considerando amostragem complexa (efeito delineamento de dois), obteve-se o número de 597 alunos. De modo a permitir a operacionalização da pesquisa, optou-se por uma amostra com aproximadamente o mesmo número de alunos em cada escola $(\mathrm{n}=48)$, com 30 escolas a serem avaliadas. Os estabelecimentos foram sorteados a partir de uma lista das escolas estaduais da cidade que ofereciam ensino a partir do $6^{\circ}$ ano do ensi- no fundamental até o ensino médio no ano 2014 ( $\mathrm{n}=167)$, sendo excluídas as escolas de educação especial ou indígena $(n=3)$.

Para a avaliação do entorno, optou-se por um mapeamento in loco da área contemplada, em área circular com raio de $250 \mathrm{~m}$, tomando como ponto de referência a escola sorteada, o que equivale a uma área de $196.350 \mathrm{~m}^{2}$ e aproximadamente dez minutos de caminhada no perímetro. As ruas dentro da área foram percorridas a pé. O mapeamento teve como objetivo a identificação dos estabelecimentos que comercializavam algum tipo de bebida alcoólica, os quais foram posteriormente convidados a participar da pesquisa.

\section{Instrumento}

Foi desenvolvido um instrumento pelas pesquisadoras a partir da revisão da literatura ${ }^{15-33}$, sendo avaliados os seguintes aspectos: tipos de estabelecimentos que comercializavam alguma bebida alcoólica, horário de atendimento, tipos de bebidas ofertadas segundo os grupos considerados pela OMS (cerveja, vinho e destilados) ${ }^{33}$, preços e tamanhos correspondentes, existência de propaganda de bebida alcoólica e de cartaz ou adesivo indicando a proibição da venda a menores de 18 anos.

Os questionários foram aplicados nos estabelecimentos que estavam em funcionamento no horário do turno escolar das turmas sorteadas para fazer parte da pesquisa em cada escola. Quando as informações não puderam ser obtidas apenas pela observação, o instrumento foi aplicado aos responsáveis dos estabelecimentos (supervisor ou proprietário).

\section{Variáveis}

As variáveis relacionadas aos estabelecimentos, tipos de bebidas, doses e preços foram obtidas por meio de um questionário que permitiu identificar as seguintes informações: 1) tipo de local que comercializa bebidas alcoólicas; 2) venda de bebidas alcoólicas (não, sim); 3) tipos de bebidas alcoólicas comercializadas (cerveja, vinho, destilados, drinks preparados ou prontos, bebidas tipo ice, outros); 4) menor preço de dose padrão para cerveja, vinho e destilados; 5) existência de algum tipo de propaganda de bebidas alcoólicas (não, sim); e 6) presença de aviso "Venda proibida a menores de 18 anos" (não, sim).

As características do entorno escolar e seus estabelecimentos comerciais foram definidas 
como variáveis dependentes, sendo mensuradas por meio dos seguintes indicadores: 1) para o entorno escolar - densidade de estabelecimentos no entorno, definida como o total de locais que comercializavam algum tipo de bebida alcoólica; 2) para os estabelecimentos que vendiam álcool - menor preço encontrado para cada tipo de bebida alcoólica e tamanho correspondente (cerveja, vinho, destilado, drinks preparados ou "batidinhas prontas" e bebidas ice), horas de atendimento ao público, menor preço e dose padrão (baseada no guia de doses padrão para cerveja, vinho e destilados da OMS - cerveja $30 \mathrm{ml}$; vinho $100 \mathrm{ml}$ e destilado $30 \mathrm{ml}^{33}$.

Também se investigou a presença de aviso "Venda proibida a menores de 18 anos" e propagandas de bebidas alcoólicas. Considerou-se propaganda por meio de decoração, preço e patrocínio.

A variável independente nas análises foi a renda domiciliar per capita do Censo de 2010. No programa QGIS, versão 2.14, foi realizada a seleção dos setores que estavam contidos em cada entorno escolar avaliado, em seguida calculouse a renda média nominal desses setores. Para a apresentação dos resultados optou-se por dividir a renda em tercis: renda baixa ( $1^{\circ}$ tercil - R $\$$ 439,70 a $\mathrm{R} \$ 853,00$ ); renda média (20 tercil R\$ 854,00 a $\mathrm{R} \$ 2.104,60$ ); e renda alta (30 tercil - R\$ $2.104,70$ a R\$ 4.645,60).

A distância da escola até o marco zero da cidade (centro) foi estimada no programa QGIS, em quilômetros, e categorizada em tercis: 1,6-5,6; $5,7-8,2 ; 8,3$ ou mais. A distância do estabelecimento que comercializava álcool mais próximo da escola foi calculada do mesmo modo (categorias: $0-100 \mathrm{~m}$; $101-250 \mathrm{~m} ; 251 \mathrm{~m}$ ou mais).

\section{Análise dos dados}

Foram realizadas análises descritivas por meio do cálculo de médias, desvio-padrão e valores mediano, mínimo e máximo da distribuição das variáveis contínuas. A distribuição das características dos estabelecimentos foi feita individualmente, e a dos estabelecimentos em relação às escolas foi apresentada por meio do cálculo das frequências absolutas e relativas.

Para identificar diferenças nas características dos estabelecimentos (variáveis dependentes) em relação à renda do entorno escolar foram estimados os coeficientes de regressão (beta) por meio de modelos multiníveis mistos. O primeiro nível foi representado por cada estabelecimento e o segundo nível pela escola (ou aqueles esta- belecimentos localizados no raio de $250 \mathrm{~m}$ de cada escola). Foram calculados os coeficientes de correlação intraclasse (ICC), que representam o percentual de variância atribuída ao segundo nível da análise, no caso as escolas. A análise multinível foi adotada em função da estrutura dos dados coletados: estabelecimentos no entorno de escolas. Os estabelecimentos representam a unidade de nível mais baixo, e as escolas o segundo nível. Espera-se que a proximidade dos estabelecimentos em cada entorno escolar faça com que compartilhem uma série de características que o diferencie dos demais entornos, e que gere uma relação de dependência entre os resultados. A independência das informações é um pressuposto da regressão tradicional, e que é violado no tipo de informações empregadas para o presente estudo. A análise multinível, ao contrário da regressão tradicional, permite considerar essa estrutura dos dados nas análises. Também foi investigada a densidade dos diferentes tipos de estabelecimentos (bares, restaurantes, postos de gasolina etc.) de acordo com a renda do entorno escolar.

A comparação das características dos estabelecimentos conforme a disponibilidade ou não de bebidas alcoólicas para consumo no local, bem como em relação aos diferentes tipos de estabelecimentos, foi avaliada por meio do teste do qui-quadrado de Pearson, ou do teste não paramétrico de Mann-Whitney para variáveis contínuas. As análises foram realizadas no programa STATA 12 (StataCorp, Texas-USA).

\section{Aspectos Éticos}

O projeto de pesquisa foi aprovado pelo Comitê de Ética em Pesquisa em Seres Humanos do Setor de Ciências da Saúde (CEP/SD) da Universidade Federal do Paraná e, posteriormente, encaminhado aos representantes da Superintendência de Desenvolvimento Educacional (SUDE) do Paraná para obter o parecer de viabilidade e execução da pesquisa. O consentimento dos participantes foi informado de forma verbal, explicando-lhes previamente sobre a pesquisa e seus procedimentos. Quando solicitadas, foram disponibilizadas informações para contato.

\section{Resultados}

Nas 30 escolas sorteadas, foram localizados 212 estabelecimentos comerciais, sete se recusaram a participar da pesquisa e cinco não foram avaliados devido à localização em área de risco. Dos 
200 estabelecimentos examinados, 66,0\% comercializavam algum tipo de bebida alcoólica e $40,2 \%$ também vendiam cigarro. No entorno de cinco escolas não foi encontrado nenhum tipo de estabelecimento que comercializava álcool, as outras 25 contavam com pelo menos um ponto de venda de álcool no entorno, e 11 tinham um ponto de venda localizado a 100 metros ou menos de distância da escola.

Dos estabelecimentos que comercializavam álcool, os pontos de venda para consumo fora do local representaram $60,6 \%$ e foram localizados em $86,7 \%$ dos entornos. Nessa categoria, os mercados de bairro $(16,1 \%)$ e os postos de gasolina (10,5\%) tiveram maior presença, e metade das escolas contavam com esses tipos de estabelecimentos em seus entornos. Na categoria de pontos de venda para consumo no local, o restaurante foi aquele encontrado com mais frequência, sendo que $28,0 \%$ comercializavam álcool e metade das escolas contava com pelo menos um estabelecimento desse tipo no entorno avaliado. Do total de estabelecimentos que comercializava álcool, $29,5 \%$ tinham expostas propagandas de bebidas alcoólicas, sendo as sobre promoções e preços as mais comuns, seguidas de patrocínio (mesas, cadeiras, cascos e geladeiras com logos da marca). Em 13,6\% dos pontos de venda de álcool foram encontrados o aviso "Proibida a venda de bebidas alcoólicas a menores de 18 anos” (Tabela 1).

Em relação aos preços, entre as bebidas alcoólicas com menor preço da dose padrão destacou-se o destilado ( $\mathrm{R} \$ 2,23$ ), seguido do vinho ( $\mathrm{R} \$ 2,86)$, mas também foram as bebidas com maior variação de preços entre os estabelecimentos (Tabela 2).

Quanto à distância, a média entre as escolas estaduais sorteadas em relação ao centro da cidade foi de 7,28 km e variou de $1,60 \mathrm{~km}$ a $18,17 \mathrm{~km}$.

A Tabela 3 apresenta as características dos pontos de venda para consumo de bebida alcóolica no local e dos pontos de venda para consumo fora do local. Houve maior variedade de tipos de bebidas alcoólicas ofertadas nos pontos de venda para consumo fora do local, assim como menores preços de dose padrão de vinho (P50: 1,5; p = 0,000) e destilados (P50: 0,3; p = 0,000), menos da metade dos valores praticados pelos estabelecimentos de consumo dentro (p50: 4,0; e p50: 3,$0 ; \mathrm{p}=0,000$, respectivamente).

$\mathrm{Na}$ Tabela 4, observa-se menor frequência de mercados de bairro e bares nos entornos com maior renda. Já estabelecimentos como cafés $\beta=$ 0,05 (IC 95\%: 0,01; 0,08) e restaurantes $\beta=0,21$ (IC 95\%: 0,04; 0,36) foram mais frequentes em setores com maior renda. Do mesmo modo, esses entornos apresentaram maiores preços de doses padrão para os três tipos de bebidas avaliadas.

\section{Discussão}

O mapeamento in loco dos entornos das escolas delimitadas na pesquisa possibilitou a coleta de informações sobre os pontos de venda de álcool para, posteriormente, avaliar a influência da renda dos setores censitários na distribuição desses pontos e quanto a suas características.

Do total de estabelecimentos avaliados nos entornos, a maioria comercializava álcool e estava em funcionamento no período de aula. Em poucas escolas não foi encontrado nenhum ponto de venda de bebida alcoólica $(n=5)$, o que pode estar relacionado à localização das escolas, pois ficam a maiores distâncias do centro da cidade, considerando que a média de distância entre as escolas sorteadas e o marco zero foi de 7,28 $\mathrm{km}$, e ainda com áreas de plantação, fábricas ou hipódromo nas redondezas.

$\mathrm{Na}$ análise geral dos estabelecimentos foi possível observar maiores preços de doses padrão e menor variedade de bebidas alcoólicas ofertadas nos entornos de alta renda. Da mesma forma, estabelecimentos como mercados de bairro e bares (popularmente chamados de "botecos", que nesta pesquisa se caracterizaram por ter uma infraestrutura mais improvisada) foram menos frequentes nesses entornos; já os cafés e as bancas de jornal foram mais comuns. A menor disponibilidade comercial de bebida alcoólica tem sido associada a padrões de consumo moderados e a bairros com alta renda ${ }^{20,21,32}$, porém esses padrões de consumo mudam quando renda individual, gênero e etnia são considerados ${ }^{37}$.

Outro dado que chamou atenção é que apenas $13,6 \%$ dos estabelecimentos que comercializavam álcool apresentaram aviso de "Venda proibida de bebida alcoólica a menores de 18 anos", a maioria deles da categoria de pontos de venda para consumo fora do local, como supermercados e postos de gasolina/lojas de conveniência. Isso sugere a necessidade de reforçar a visibilidade da Lei no 8.069/1990 - Estatuto da Criança e do Adolescente, que proíbe a venda a menores de 18 anos, e a fiscalização da Lei Estadual $15.443 / 2007$, na qual se dispõe sobre a afixação de cartazes constando a expressão mencionada nos locais que comercializam bebidas alcoólicas ${ }^{34,35}$.

No aspecto relativo às doses padrão de cerveja, vinho e destilado, os achados mostraram menor preço para o vinho, seguido dos destila- 
Tabela 1. Características dos estabelecimentos em relação à disponibilidade de bebidas alcoólicas avaliados nos entornos de escolas estaduais*. Curitiba, Paraná, 2016-2017.

\begin{tabular}{|c|c|c|c|c|}
\hline & \multicolumn{2}{|c|}{$\begin{array}{l}\text { Estabelecimentos } \\
\quad(n=200)\end{array}$} & \multicolumn{2}{|c|}{$\begin{array}{l}\text { Escolas } \\
(\mathbf{n}=\mathbf{3 0})\end{array}$} \\
\hline & $\mathbf{N}$ & $\%$ & n & $\%$ \\
\hline \multicolumn{5}{|c|}{$\begin{array}{l}\text { Estabelecimento com cartaz de venda proibida a menores de } 18 \text { anos" } \\
(\mathrm{n}=132)\end{array}$} \\
\hline Sim & 18 & 13,6 & 11 & 36,7 \\
\hline Não & 114 & 86,4 & 19 & 63,3 \\
\hline Pontos de venda de álcool para consumo no local & 5 & 3,8 & 3 & 10,0 \\
\hline Pontos de venda de álcool para consumo fora do local & 13 & 9,9 & 10 & 33,3 \\
\hline \multicolumn{5}{|c|}{ Período de funcionamento dos pontos de venda de álcool ${ }^{\star *}(\mathrm{n}=123)$} \\
\hline Manhã & 1 & 0,8 & 1 & 3,3 \\
\hline Tarde & 1 & 0,8 & 1 & 3,3 \\
\hline Manhã e tarde & 32 & 24,2 & 17 & 56,7 \\
\hline Tarde e noite & 5 & 3,8 & 5 & 16,7 \\
\hline Manhã, tarde e noite & 84 & 63,6 & 23 & 76,7 \\
\hline \multicolumn{5}{|l|}{ Ponto de venda de álcool mais próximo ${ }^{\star * \star}(\mathrm{m})(\mathrm{n}=130)$} \\
\hline $0-100$ & 20 & 15,4 & 11 & 36,7 \\
\hline $101-250$ & 92 & 70,8 & 10 & 33,3 \\
\hline 251 e mais & 18 & 13,8 & 9 & 30,0 \\
\hline
\end{tabular}

* Considerou-se como entorno escolar, a área dentro de buffer circular com raio de $250 \mathrm{~m}$, tomando como centro a escola; ${ }^{\star *}$ diferenças nos $\mathrm{n}$ totais se devem a perguntas não respondidas; ${ }^{\star * *}$ diferença com o $\mathrm{n}$ total deve-se à perda dos endereços.

Fonte: Elaborado pelos autores.

Tabela 2. Descrição das variáveis relacionadas ao entorno escolar. Curitiba, Paraná, 2016-2017.

\begin{tabular}{|c|c|c|c|c|c|}
\hline Variáveis & Fonte & Média & Mediana & $\begin{array}{l}\text { Desvio } \\
\text { padrão }\end{array}$ & Min.-máx. \\
\hline \multicolumn{6}{|l|}{ Entorno Escolar ${ }^{\mathrm{a}}$} \\
\hline $\begin{array}{l}\text { Estabelecimentos nas áreas das } \\
\text { escolas }\end{array}$ & Mapeamento & 6,6 & 5 & 5,4 & $0-18$ \\
\hline Estabelecimentos avaliados & Mapeamento & 6,3 & 5 & 5,1 & $0-18$ \\
\hline Pontos de venda de álcool & Mapeamento & 1,4 & 1 & 1,8 & $0-7$ \\
\hline Pontos de venda de cigarro & Coleta primária & 2.0 & 2 & 1,8 & $0-7$ \\
\hline Pontos de venda de álcool e cigarro & Coleta primária & 0,9 & 1 & 1,1 & $0-4$ \\
\hline $\begin{array}{l}\text { Preço da dose padrão de cerveja } \\
\text { mais barata }\end{array}$ & Coleta primária & 3,59 & 2,83 & 2,45 & $1,50-5,38$ \\
\hline $\begin{array}{l}\text { Preço da dose padrão de vinho } \\
\text { mais barato }\end{array}$ & Coleta primária & 2,86 & 1,70 & 3,22 & $0,35-23,00$ \\
\hline $\begin{array}{l}\text { Preço da dose padrão de destilado } \\
\text { mais barato }\end{array}$ & Coleta primária & 2,23 & 0,38 & 4,21 & $0,12-22,00$ \\
\hline $\begin{array}{l}\text { Rendimento Domiciliar per capita } \\
(\mathrm{R} \$)^{*}\end{array}$ & Censo 2011 & $2.916,86$ & $2.116,57$ & $2.193,35$ & $843,00-8.088,52$ \\
\hline Distância do marco zero da cidade & Mapeamento & $7.274,90$ & $6.838,25$ & $4.074,61$ & $1.603,10-18.171,78$ \\
\hline
\end{tabular}

Fonte: Elaborado pelos autores.

dos. Resultados condizentes com a pesquisa da Faculdade Latino-Americana de Ciências Sociais (FLACSO) na qual destaca-se que, em regiões com colonização europeia mais recente, há uma maior produção dessa bebida (mesmo que de forma caseira), o que diminui o custo, e o vinho 
Tabela 3. Descrição das características das categorias de pontos de venda de álcool. Curitiba, Paraná, 2016-2017.

\begin{tabular}{|c|c|c|}
\hline Variáveis & $\begin{array}{l}\text { Pontos de venda de álcool } \\
\text { para consumo no local }\end{array}$ & $\begin{array}{l}\text { Pontos de venda de álcool } \\
\text { para consumo fora do local }\end{array}$ \\
\hline Propaganda de bebida alcoólica ${ }^{\mathrm{a}}$ - n (\%) & $18(25,0)$ & $23(19,7)$ \\
\hline Aviso de enda proibida a menores de & $4(5,6)$ & $12(10,3)$ \\
\hline \multicolumn{3}{|l|}{$18 \operatorname{anos}^{\mathrm{a}}-\mathrm{n}(\%)$} \\
\hline 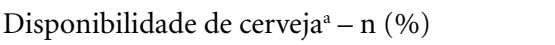 & $51(70,8)$ & $74(63,3)$ \\
\hline Disponibilidade de vinho $^{\mathrm{a}}-\mathrm{n}(\%)$ & $26(36,1)$ & $75(55,6)^{*}$ \\
\hline Disponibilidade de destilados ${ }^{\mathrm{a}}-\mathrm{n}(\%)$ & $28(38,9)$ & $62(53,0)^{*}$ \\
\hline Disponibilidade de drink preparado ${ }^{\mathrm{a}}-\mathrm{n}(\%)$ & $28(38,9)$ & $41(35,0)$ \\
\hline Disponibilidade de bebidas ${ }^{\mathrm{a}}$ ice $-\mathrm{n}(\%)$ & $6(8,3)^{*}$ & $28(23,9)^{*}$ \\
\hline $\begin{array}{l}\text { Tipos de bebidas ofertadas (número) })^{\mathrm{b}}-\mathrm{p} 50 \\
\text { (p25-p75) }\end{array}$ & $1,5(0-4,0)$ & $3,0(0-4,0)^{*}$ \\
\hline $\begin{array}{l}\text { Preço da dose padrão mais barata de } \\
\text { cerveja }{ }^{\mathrm{b}}-\mathrm{p} 50(\mathrm{p} 25-\mathrm{p} 75)\end{array}$ & $3,8(2,8-6,5)^{*}$ & $2,4(1,9-2,8)^{\star}$ \\
\hline $\begin{array}{l}\text { Preço da dose padrão mais barata de } \\
\text { vinho }^{\mathrm{b}}-\mathrm{p} 50(\mathrm{p} 25-\mathrm{p} 75)\end{array}$ & $4,0(2,5-6,7)^{\star}$ & $1,5(1,2-1,9)^{\star}$ \\
\hline $\begin{array}{l}\text { Preço da dose padrão mais barata de } \\
\text { destilado }^{\mathrm{b}}-\mathrm{p} 50(\mathrm{p} 25-\mathrm{p} 75)\end{array}$ & $3,0(2,0-7,3)^{*}$ & $0,3(0,2-0,4)^{*}$ \\
\hline Horas de atendimento ao público ${ }^{\mathrm{b}}$ & $10,0(3,5-12,0)$ & $11,5(10,0-12,5)^{*}$ \\
\hline
\end{tabular}

${ }^{*} \mathrm{p}<0,05 ;{ }^{a}$ teste qui-quadrado ; ${ }^{\mathrm{b}}$ teste Mann-Whitney.

Fonte: Elaborado pelos autores.

comum (ou de mesa) pode ser considerado um suplemento alimentar, sendo consumido principalmente pela população de menor renda ${ }^{36}$. $\mathrm{Na}$ realização da pesquisa foi possível observar a oferta de vinhos caseiros, vendidos em maior volume (geralmente $1.000 \mathrm{ml}$ ) e com preços menores quando comparados aos vinhos finos (ou de maior qualidade) ou mesmo com os vinhos produzidos na região.

$\mathrm{Na}$ literatura internacional, a tipologia pontos de venda de álcool para consumo no local e fora do local é a mais utilizada nas pesquisas que estudam a relação entre a oferta de álcool por meio da densidade de pontos de venda e consu$\mathrm{mo}^{11,15}$. No presente estudo, observou-se diferenças em relação a menores preços de doses padrão, ao horário de atendimento ao público, que são mais extensos, e à maior variedade de bebidas alcoólicas em pontos de venda para consumo fora do local. Esses aspectos podem favorecer o alcance, pois sabe-se que os adolescentes têm acesso às bebidas alcoólicas principalmente por meio de terceiros (fontes de acesso sociais), mas também por fontes comerciais, exemplo disso é que no Brasil as fontes referidas são festas, amigos, mercados e família, respectivamente ${ }^{2}$.

Estudos internacionais mostraram que os locais com menores vantagens socioeconômicas tendem a ter maior densidade de pontos de venda de álcool ${ }^{20,21,32}$ e que os pontos de venda para consumo no local estão mais fortemente associados ao alto consumo de álcool por adolescentes $^{22,26,37}$. No contexto brasileiro, não há pesquisas que tenham estudado essas dimensões.

Nos entornos escolares avaliados, observouse que os pontos de venda para consumo no local foram mais frequentes naqueles categorizados no tercil de maior renda. Adicionalmente, restaurantes e cafés tiveram maior presença nos setores de alta renda, e os bares foram mais numerosos nos setores de menor renda. Os restaurantes tiveram maior representatividade, na análise geral de estabelecimentos, na categoria de pontos de venda para consumo no local. Por outro lado, observou-se maior frequência de bares e mercados de bairro em entornos de menor renda. As características desses estabelecimentos, como horário de atendimento mais extenso, maior variedade de tipos de bebida alcoólica e menores preços de doses padrão (quando comparados aos restaurantes e cafés), tornam-se importantes para o desenvolvimento de estratégias e intervenções de saúde, pois de acordo com a revisão realizada por Collins ${ }^{37}$, as desvantagens econômicas do local foram associadas ao consumo excessivo de álcool e a consequências negativas entre as pessoas que bebem.

O presente trabalho apresenta algumas limitações, como o tamanho da área avaliada, o que dificulta a comparação com estudos internacio- 
Tabela 4. Associação entre as características relativas a disponibilidade comercial de bebidas alcoólicas e renda dos entornos de escolas estaduais $(n=30)$. Curitiba, Paraná, 2016-2017.

\begin{tabular}{|c|c|c|c|c|c|}
\hline & \multicolumn{5}{|c|}{ Rendimento domiciliar per capita do entorno escolar $2010(\mathrm{R} \$)$} \\
\hline & $1^{\circ}$ tercil & $2 \circ$ tercil & $3^{\circ}$ tercil & & \\
\hline & $\mathrm{R} \$ 439,7-\mathrm{R} \$ 853$ & $\mathrm{R} \$ 854-\mathrm{R} \$ 2.104,6$ & $\mathrm{R} \$ 2.104,7-\mathrm{R} \$ 4.645,6$ & Valor de & ICC \\
\hline Variáveis ${ }^{*}$ & Coef. (IC 95\%) & Coef. (IC 95\%) & Coef. (IC 95\%) & $\mathbf{p}^{\mathrm{a}}$ & \\
\hline Venda de bebida alcoólica & Ref. & $-0,02(-0,19-0,13)$ & $-0,15(-0,30-0,00)$ & 0,000 & 0,000 \\
\hline $\begin{array}{l}\text { Tipos de bebidas ofertadas } \\
\text { (número) }\end{array}$ & Ref. & $-0,38(-1,02-0,26)$ & $-0,57(-1,18-0,04)$ & 0,000 & 0,065 \\
\hline $\begin{array}{l}\text { Pontos de venda de álcool } \\
\text { para consumo no local }\end{array}$ & Ref. & $0,15(-0,03-0,33)$ & $0,20(0,02-0,37)$ & 0,021 & 0,028 \\
\hline $\begin{array}{l}\text { Pontos de venda de álcool } \\
\text { para consumo fora do local }\end{array}$ & Ref. & $-0,06(-0,23-0,10)$ & $-0,11(-0,28-0,05)$ & 0,160 & 0,000 \\
\hline $\begin{array}{l}\text { Propaganda de bebida } \\
\text { alcoólica }\end{array}$ & Ref. & $-0,01(-0,17-0,16)$ & $0,11(-0,05-0,27)$ & 0,193 & 0,055 \\
\hline $\begin{array}{l}\text { Aviso de venda proibida a } \\
\text { menores de } 18 \text { anos }\end{array}$ & Ref. & $0,01(-0,08-0,10)$ & $0,08(-0,01-0,17)$ & 0,106 & 0,024 \\
\hline $\begin{array}{l}\text { Preço da dose padrão mais } \\
\text { barata de cerveja }\end{array}$ & Ref. & $0,51(-0,76-1,78)$ & $2,58(1,31-3,84)$ & 0,000 & 0,224 \\
\hline $\begin{array}{l}\text { Preço da dose padrão mais } \\
\text { barata de vinho }\end{array}$ & Ref. & $0,77(-0,63-2,18)$ & $3,18(1,68-4,69)$ & 0,000 & 0,000 \\
\hline $\begin{array}{l}\text { Preço da dose padrão mais } \\
\text { barata de destilado }\end{array}$ & Ref. & $0,39(-1,66-2,43)$ & $4,64(2,60-6,67)$ & 0,000 & 0,075 \\
\hline $\begin{array}{l}\text { Supermercados/ } \\
\text { hipermercados }\end{array}$ & Ref. & $-0,00^{* *}(-0,03-0,03)$ & $0,03(-0,00-0,06)$ & 0,069 & 0,000 \\
\hline Mercados de bairro & Ref. & $-0,03(-0,14-0,09)$ & $-0,16(-0,26--0,05)$ & 0,005 & 0,000 \\
\hline Restaurantes & Ref. & $0,09(-0,08-0,25)$ & $0,21(0,04-0,36)$ & 0,011 & 0,016 \\
\hline Bares & Ref. & $-0,02(-0,11-0,08)$ & $-0,13(-0,22-0,03)$ & 0,007 & 0,009 \\
\hline Cafés & Ref. & $-0,00^{* *}(-0,04-0,04)$ & $0,05(0,01-0,08)$ & 0,025 & 0,000 \\
\hline $\begin{array}{l}\text { Padarias com setor de } \\
\text { conveniência }\end{array}$ & Ref. & $-0,05(-0,13-0,04)$ & $-0,05(-0,13-0,03)$ & 0,193 & 0,000 \\
\hline $\begin{array}{l}\text { Postos de gasolina/loja de } \\
\text { conveniência }\end{array}$ & Ref. & $0,02(-0,09-0,13)$ & $0,05(-0,05-0,16)$ & 0,331 & 0,021 \\
\hline Sacolão & Ref. & $-0,01(-0,08-0,06)$ & $-0,05(-0,11-0,20)$ & 0,178 & 0,000 \\
\hline Distribuidoras de bebidas & Ref. & $-0,05(-0,13-0,03)$ & $-0,04(-0,12-0,04)$ & 0,273 & 0,000 \\
\hline Outros & Ref. & $0,05(-0,07-0,17)$ & $-0,01(-0,13-0,11)$ & 0,889 & 0,000 \\
\hline
\end{tabular}

Fonte: Elaborado pelos autores.

nais e restringe a generalização do estudo, uma vez que a amostra foi calculada com base na representatividade de alunos nas escolas estaduais, e não do total de escolas. Por outro lado, observou-se que as escolas sorteadas se distribuíram em todas as regionais do município e em locais com ampla variação socioeconômica. O principal avanço deste estudo em relação aos encontrados na literatura sobre pontos de comercialização de bebidas alcóolicas está nos procedimentos para identificação e caracterização desses estabe- lecimentos, com auditagem dentro dos locais, levando em consideração que estavam situados nas proximidades de escolas estaduais. Em um cenário em que é cada vez maior o número de adolescentes que consomem álcool, e com o primeiro contato sendo cada vez mais precoce ${ }^{2,38}$, conhecer as características do ambiente físico (escolar ou residencial) e do comércio torna-se crucial para a formulação de políticas regulatórias de álcool.

Até o momento de conclusão da presente pesquisa não foram encontrados estudos que carac- 
terizassem o entorno escolar da rede de ensino estadual em Curitiba com relação à oferta de bebidas alcoólicas. As observações da pesquisa refletem os achados obtidos localmente. No entanto, outros estudos mais amplos se fazem necessários.

\section{Conclusão}

Os achados da pesquisa sugerem maior frequência de mercados de bairro e bares nos entornos localizados em setores de menor renda, e maior variedade e número de estabelecimentos nos entornos com renda mais elevada.

Em alguns entornos avaliados, estabelecimentos comercializavam bebidas alcoólicas a 100 metros ou menos das escolas, e muitos em horário de aula. Dessa forma, destaca-se a importância de repensar as leis de zoneamento comercial ou a implementação de distâncias mínimas entre estabelecimentos e escolas como alternativas para reduzir o acesso às bebidas alcoólicas pelos adolescentes no entorno escolar.

Por fim, destaca-se a necessidade de maiores esforços para ampliar a visibilidade e a fiscalização da lei que proíbe a venda de álcool a menores de idade, pois menos de um terço dos estabelecimentos avaliados nas proximidades das escolas apresentavam o cartaz. Sugere-se também a realização de intervenções educativas para os donos e atendentes dos estabelecimentos.

\section{Colaboradores}

LMS Cardoza e DA Hofelmann desenharam o estudo. LMS Cardoza e DS Santos coletaram os dados. DA Hofelmann conduziu a análise estatística em colaboração com DS Santos e LMS Cardoza. LMS Cardoza escreveu a primeira versão do manuscrito e todos os autores contribuíram e aprovaram a versão final.

\section{Referências}

1. Universidade Federal de São Paulo (UNIFESP). II Levantamento nacional de álcool e drogas (LENAD) - 2012. São Paulo: INPAD/UNIAD/UNIFESP; 2014.

2. Instituto Brasileiro de Geografia e Estatística (IBGE). Pesquisa nacional de saúde do Escolar 2015. Rio de Janeiro: IBGE; 2016.

3. World Health Organization (WHO). Global status report on alcohol and health 2014. Geneva: WHO; 2014.

4. Freitas D, Rodrigues CS, Yagui CM, Carvalho RST, Marchi-Alves LM. Fatores de risco para hipertensão arterial entre estudantes do ensino médio. Acta Paulista de Enfermagem 2012; 25(3):430-434.

5. Strauch ES, Pinheiro RT, Silva RA, Horta BL. Uso de álcool por adolescentes: estudo de base populacional. Rev Saude Publica 2009; 43(4):647-655.

6. Malta DC, Andreazzi MAR, Oliveira-Campos M, Andrade SSCA, Sá NNB, Moura L, Dias AJ, Crespo CD, Silva JB. Trend of the risk and protective factors of chronic diseases in adolescents, National Adolescent School-based Health Survey (PeNSE 2009 e 2012) Rev Bras Epidemiol 2014; 17(1):77-91.

7. Laranjeira R, Hinkly D. Evaluation of alcohol outlet density and its relation with violence. Rev Saude Publica 2002; 36(4):455-461.

8. Duailibi S, Laranjeira R. Políticas públicas relacionadas às bebidas alcoólicas. Rev Saude Publica 2007; 41(5):839-848. 
9. Morrison C, Ponicki WR, Gruenewald PJ, Wiebe DJ, Smith K. Spatial relationships between alcohol-related road crashes and retail alcohol availability. Drug Alcohol Depend 2016; 1(162):241-244.

10. Gmel G, Holmes J, Studer J. Are alcohol outlet densities strongly associated with alcohol-related outcomes? A critical review of recent evidence. Drug Alcohol Rev 2015; 35(1):40-54.

11. Larsen $\mathrm{K}$, To T, Irving HM, Boak A, Hamilton HA, Mann RE,Schwartz R, Faulkner G. Smoking and binge-drinking among adolescents, Ontario, Canada: does the school neighbourhood matter? Health Place 2017; 47:108-114.

12. Henriksen L, Feighery EC, Schleicher NC, Cowling DW, Kline RS, Fortmann SP. Is adolescent smoking related to the density and proximity of tobacco outlets and retail cigarette advertising near schools? Prev Med (Baltim) 2008; 47(2): 210-214.

13. McCarthy WJ, Mistry R, Lu Y, Patel M, Zheng H, Dietsch B. Density of tobacco retailers near schools: effects on tobacco use among students. Am J Public Health 2009; 99(11):2006-2013.

14. Scully M, McCarthy M, Zacher M, Warne C, Wakefield M, White V. Density of tobacco retail outlets near schools and smoking behaviour among secondary school students. Aust N Z J Public Health 2013; 37(6):574-578.

15. Bryden A, Roberts B, McKee M, Petticrew M. A systematic review of the influence on alcohol use of community level availability and marketing of alcohol. Health Place 2012; 18(2):349-357.

16. Reboussin BA, Preisser JS, Song E-Y, Wolfson M. Geographic clustering of underage drinking and the influence of community characteristics. Drug Alcohol Depend 2010; 106(1):38-47.

17. Botticello AL. School contextual influences on the risk for adolescent alcohol misuse. Am J Community Psychol 2009; 43(1):85-97.

18. Song E-Y, Reboussin BA, Foley KL, Kaltenbach LA, Wagoner KG, Wolfson M. Selected community characteristics and underage drinking. Subst Use Misuse 2009; 44(2):179-194.

19. Ennett ST, Flewelling RL, Lindrooth RC, Norton EC. School and neighborhood characteristics associated with school rates of alcohol, cigarette, and marijuana use. J Health Soc Behav 1997; 38(1):55-71.

20. Shortt NK, Tisch C, Pearce J, Mitchell R, Richardson EA, Hill S, Collin J. A cross-sectional analysis of the relationship between tobacco and alcohol outlet density and neighbourhood deprivation. BMC Public Health 2015; 15(1):1014.

21. Rossheim ME, Thombs DL, Wagenaar AC, Xuan Z, Aryal S. High alcohol concentration products associated with poverty and State alcohol policies. Am J Public Health 2015; 105(9):1886-1892.

22. Kuntsche E, Kuendig H, Gmel G. Alcohol outlet density, perceived availability and adolescent alcohol use: a multilevel structural equation model. $J$ Epidemiol Community Health 2008; 62(9):811-816.

23. Young R, Macdonald L, Ellaway A. Associations between proximity and density of local alcohol outlets and alcohol use among Scottish adolescents. Health Place 2013; 19(100):124-130.
24. Milam AJ, Furr-Holden CDM, Cooley-Strickland MC, Bradshaw CP, Leaf PJ. Risk for exposure to alcohol, tobacco, and other drugs on the route to and from school: the role of alcohol outlets. Prev Sci 2014; 15(1):12-21.

25. Rowland B, Toumbourou JW, Satyen L, Tooley G, Hall J, Livingston M, Willians J. Associations between alcohol outlet densities and adolescent alcohol consumption: a study in Australian students. Addict Behav 2014; 39(1):282-288.

26. Shih RA, Mullins L, Ewing BA, Miyashiro L, Tucker JS, Pedersen ER, Miles JN, D'Amico DJ. Associations between neighborhood alcohol availability and young adolescent alcohol use. Psychol Addict Behav 2015; 29(4):950-959.

27. De Boni R, Pechansky F, Silva PLN, Vasconcellos MTL, Bastos FI. Is the prevalence of driving after drinking higher in entertainment areas? Alcohol Alcohol. 2013; 48(3):356-362.

28. Vieira DL, Ribeiro M, Romano M, Laranjeira R. Alcohol and adolescents: study to implement municipal policies. Rev Saude Publica 2007; 41(3):396-403.

29. Casswell S. Commentary on White et al. (2018): decrease in adolescent drinking linked to direct and indirect effect of alcohol control policies. Addiction 2018; 113(6):1043-1044.

30. Warren JC, Smalley KB, Barefoot KN. Perceived ease of access to alcohol, tobacco and other substances in rural and urban US students. Rural Remote Health 2015; $15(4): 3397$.

31. Instituto Paranaense de Desenvolvimento Econômico e Social (Ipardes). Perfil avançado do município de Curitiba. Curitiba: Ipardes; 2017.

32. Instituto Brasileiro de Geografia e Estatística (IBGE). Infografia de Curitiba. Curitiba: IBGE; 2016.

33. World Health Organization (WHO). Self-help strategies: for cutting down or stopping substance use, 2010. Geneva: WHO; 2010.

34. Paraná. Lei 15.443, 15 de Janeiro de 2007. [acessado 2007 Jan 15] Disponível em: http://www.legislacao. pr.gov.br/legislacao/pesquisarAto.do?action=exibir\&codAto $=1665 \&$ indice $=1 \&$ total Registros $=1$.

35. Brasil. Lei ${ }^{\circ} 8.069,13$ de julho de 1990. Considerase criança, para os efeitos desta Lei, a pessoa até doze anos de idade incompletos, e adolescente aquela entre doze e dezoito anos de idade. Diário Oficial da União 1990; 16 jul.

36. Truong KD, Sturm R. Alcohol environments and disparities in exposure associated with adolescent drinking in California. Am J Public Health 2009; 99(2):264-270.

37. Collins SE. Associations Between Socioeconomic Factors and Alcohol Outcomes. Alcohol Res 2016; 38(1):83-94.

38. Instituto Brasileiro de Geografia e Estatística (IBGE). Pesquisa Nacional de Saúde do Escolar 2013. Rio de Janeiro: IBGE; 2015.

Artigo apresentado em 19/07/2018

Aprovado em 16/09/2019

Versão final apresentada em 18/09/2019

Editores-chefes: Romeu Gomes, Antônio Augusto Moura da Silva 